PROCEEDINGS OF THE

AMERICAN MATHEMATICAL SOCIETY

Volume 137, Number 3, March 2009, Pages 877-883

S 0002-9939(08)09724-4

Article electronically published on October 15, 2008

\title{
SIMPLE ALGEBRAS OF GELFAND-KIRILLOV DIMENSION TWO
}

JASON P. BELL

(Communicated by Birge Huisgen-Zimmermann)

\begin{abstract}
Let $k$ be a field. We show that a finitely generated simple Goldie $k$-algebra of quadratic growth is noetherian and has Krull dimension 1. Thus a simple algebra of quadratic growth is left noetherian if and only if it is right noetherian. As a special case, we see that if $A$ is a finitely generated simple domain of quadratic growth, then $A$ is noetherian and by a result of Stafford every right and left ideal is generated by at most two elements. We conclude by posing questions and giving examples in which we consider what happens when the hypotheses are relaxed.
\end{abstract}

\section{INTRODUCTION}

We study finitely generated algebras of quadratic growth. Given a field $k$ and a finitely generated $k$-algebra $A$, a $k$-subspace $V$ of $A$ is called a frame of $A$ if $V$ is finite dimensional, $1 \in V$, and $V$ generates $A$ as a $k$-algebra. We say that $A$ has quadratic growth if there exist a frame $V$ of $A$ and constants $C_{1}, C_{2}>0$ such that

$$
C_{1} n^{2} \leq \operatorname{dim}_{k}\left(V^{n}\right) \leq C_{2} n^{2} \quad \text { for all } n \geq 1 \text {. }
$$

We note that an algebra of quadratic growth has GK dimension 2. More generally, the Gelfand-Kirillov dimension (GK dimension, for short) of a finitely generated $k$-algebra $A$ is defined to be

$$
\operatorname{GKdim}(A)=\limsup _{n \rightarrow \infty} \frac{\log \left(\operatorname{dim}\left(V^{n}\right)\right)}{\log n},
$$

where $V$ is a frame of $A$. While algebras of quadratic growth have GK dimension 2 , it is not the case that an algebra of GK dimension 2 necessarily has quadratic growth.

GK dimension can be viewed as a noncommutative analogue of Krull dimension in the following sense: if $A$ is a finitely generated commutative $k$-algebra, then the Krull dimension of $A$ and the GK dimension of $A$ coincide. Krull dimension can also be extended to the noncommutative setting in a natural way by considering the so-called deviation of the poset of left ideals of a ring (see McConnell and Robson [5. Chapter 6] for the actual definition of Krull dimension). Unlike GK dimension,

Received by the editors December 21, 2007, and, in revised form, February 21, 2008, and March 17, 2008.

2000 Mathematics Subject Classification. Primary 16P90; Secondary 16P40.

Key words and phrases. GK dimension, quadratic growth, simple rings, noetherian rings.

The author thanks NSERC for its generous support.

(C)2008 American Mathematical Society Reverts to public domain 28 years from publication 
however, Krull dimension does not always exist. This is one weakness of Krull dimension, but it always exists for noetherian rings. It is in this more general sense that we use the term Krull dimension in this paper. For more information on GK dimension we refer the reader to Krause and Lenagan [4].

We study simple Goldie algebras of quadratic growth. A ring $R$ is right Goldie if it satisfies the following two conditions:

(1) $R$ does not contain an infinite direct sum of nonzero right ideals;

(2) $R$ satisfies the ascending chain condition on right annihilators.

Left Goldie is defined analogously. A ring that is both left and right Goldie is called Goldie. A result of Irving and Small [3] shows that right Goldie and left Goldie are equivalent for semiprime algebras of finite GK dimension.

The Goldie condition is named after Alfred Goldie, who defined this property in his study of noncommutative localization. Just as a commutative domain has a field of fractions, a semiprime Goldie ring $R$ has a semisimple Artinian ring of quotients $Q(R)$. We note that noetherian 11 algebras are always Goldie, but there exist non-noetherian Goldie algebras; for example, a polynomial ring in infinitely many variables over a field is Goldie but not noetherian.

Our main result is the following theorem, which shows that the Goldie and noetherian properties are equivalent for simple algebras of quadratic growth.

Theorem 1.1. Let $k$ be a field and let $A$ be a finitely generated simple Goldie $k$-algebra of quadratic growth. Then $A$ is a noetherian algebra of Krull dimension 1.

A result of Jategaonkar (cf. Krause and Lenagan [4, Proposition 4.13]) shows that a domain of finite GK dimension is Goldie. Hence we get the following corollary.

Corollary 1.2. Let $k$ be a field and let $A$ be a finitely generated simple $k$-algebra of quadratic growth that is a domain. Then $A$ is a noetherian algebra of Krull dimension 1. In particular, every left and right ideal can be generated by two elements.

The fact that every right and left ideal of a simple noetherian algebra of Krull dimension one is generated by at most two elements follows from a result of Stafford [8. Another interesting corollary we obtain is the following.

Corollary 1.3. Let $A$ be a finitely generated simple algebra of quadratic growth. Then $A$ is left noetherian if and only if $A$ is right noetherian.

The outline of the paper is as follows. In section 2, we give proofs of Theorem 1.1 and Corollary 1.2. In section 3, we give some examples in which we consider what happens when various hypotheses are relaxed and give some concluding remarks.

\section{Proofs}

In this section we give the proofs of Theorem 1.1 and Corollary 1.2, We begin with a few estimates for algebras of quadratic growth.

Lemma 2.1. Let $K$ be a field, let $A$ be a finitely generated $K$-algebra with quadratic growth, and let $m$ be a positive integer. If $V$ is a finite-dimensional $K$-subspace of

\footnotetext{
${ }^{1}$ We take noetherian to mean satisfying the ascending chain condition on both left and right ideals.
} 
$A$ that generates $A$ as a $K$-algebra, then there is a positive constant $C=C(m, V)$ such that

$$
\operatorname{dim}\left(V^{n}\right)-\operatorname{dim}\left(V^{n-m}\right) \leq C n
$$

for infinitely many $n \geq 1$.

Proof. By assumption, $A$ has quadratic growth and hence there is a positive constant $C_{0}$ such that

$$
\operatorname{dim}\left(V^{n}\right) \leq C_{0} n^{2} \quad \text { for } n \text { sufficiently large. }
$$

We take

$$
C=4 C_{0} m \text {. }
$$

Suppose that there is a number $N$ such that

$$
\operatorname{dim}\left(V^{n}\right)-\operatorname{dim}\left(V^{n-m}\right)>C n
$$

for all $n>N$. Then

$$
\begin{aligned}
\operatorname{dim}\left(V^{n m+N}\right) & \geq \operatorname{dim}\left(V^{n m+N}\right)-\operatorname{dim}\left(V^{N}\right) \\
& =\sum_{j=0}^{n-1}\left(\operatorname{dim}\left(V^{N+j m+m}\right)-\operatorname{dim}\left(V^{N+j m}\right)\right) \\
& \geq \sum_{j=0}^{n-1} C(N+j m+m) \\
& =C N n+C m(n+1) n / 2 \\
& \geq 2 C_{0} n^{2} m^{2} \\
& >C_{0}(n m+N)^{2} \quad \text { (for } n \text { sufficiently large). }
\end{aligned}
$$

This is a contradiction. The result follows.

Our next lemma gives an estimate for the growth of $A / I$ when $I$ contains a regular element $a$. We recall that an element $a$ is regular if its right and left annihilators are both (0).

Lemma 2.2. Let $k$ be a field and let $A$ be a prime finitely generated $k$-algebra with quadratic growth. Let $V$ be a finite-dimensional generating subspace of $A$ that contains 1 . If $I$ is a right ideal of $A$ and I contains a regular element a, then there is a positive constant $C$ such that

$$
\operatorname{dim}\left(V^{n} / V^{n} \cap I\right) \leq C n
$$

for infinitely many $n$.

Proof. By assumption $A$ has quadratic growth and hence there is a positive constant $C_{0}$ such that

$$
\operatorname{dim}\left(V^{n}\right) \leq C_{0} n^{2} \quad \text { for } n \geq 1 .
$$

Let $I$ be a nonzero left ideal of $A$ and suppose there is a regular element $a \in I$. Then $a \in V^{m}$ for some natural number $m$. Then

$$
\begin{aligned}
\operatorname{dim}\left(V^{n} /\left(V^{n} \cap I\right)\right) & =\operatorname{dim}\left(V^{n}\right)-\operatorname{dim}\left(V^{n} \cap I\right) \\
& \leq \operatorname{dim}\left(V^{n}\right)-\operatorname{dim}\left(V^{n} \cap a A\right) \\
& \leq \operatorname{dim}\left(V^{n}\right)-\operatorname{dim}\left(a V^{n-m}\right) \\
& =\operatorname{dim}\left(V^{n}\right)-\operatorname{dim}\left(V^{n-m}\right) .
\end{aligned}
$$


By the preceding lemma there is a positive constant $C$ such that

$$
\operatorname{dim}\left(V^{n}\right)-\operatorname{dim}\left(V^{n-m}\right) \leq C n
$$

for infinitely many $n$. The result follows.

Lemma 2.3. Let $K$ be a field and let $A$ be an infinite simple affine $K$-algebra. Let $V$ be a finite-dimensional generating subspace of $A$ which contains 1 . If $I \subseteq J$ are two right ideals in $A$ and $I \neq J$, then there is $m>0$ such that

$$
\operatorname{dim}\left(V^{n} \cap J / V^{n} \cap I\right) \geq n-m
$$

for all $n \geq 0$.

Proof. Pick $a \in J \backslash I$. Without loss of generality, we may assume $J=I+a A$. Note that if $I+a V^{m} \supseteq I+a V^{m+1}$, then $I+a A \subseteq I+a V^{m}$, and hence $J / I$ is finite dimensional as a $K$-vector space. But this says that $A$ has a finite-dimensional nonzero right $A$-module, which is impossible since $A$ is infinite and simple. It follows that

$$
\operatorname{dim}\left(I+a V^{n} / I\right) \geq n
$$

for all $n \geq 0$. Since $V$ is a generating subspace, there is some $m$ such that $a \in V^{m}$. Hence

$$
\operatorname{dim}\left(J \cap V^{n+m} / I \cap V^{n+m}\right) \geq \operatorname{dim}\left(\left(I+a V^{n}\right) / I\right) \geq n .
$$

The result follows.

Proof of Theorem 1.1. Let $V$ be a finite-dimensional $K$-subspace of $A$ that generates $A$ as a $K$-algebra. By assumption $A$ has quadratic growth and hence there is a positive constant $C$ such that

$$
\operatorname{dim}\left(V^{n}\right) \leq C n^{2} \quad \text { for } n \geq 1 .
$$

Let $I$ be a nonzero right ideal. By Lemma 2.2 there is a positive constant $C=$ $C(I, V)$ such that

$$
\operatorname{dim}\left(V^{n} /\left(V^{n} \cap I\right)\right) \leq C n
$$

for infinitely many $n$. Let $m$ denote the uniform dimension of $A$ (see McConnell and Robson [5, 2.2.10]). We claim that if

$$
I=I_{0} \subseteq I_{1} \subseteq I_{2} \subseteq \cdots \subseteq I_{e}
$$

is an ascending chain of ideals in which each containment is proper, then $e \leq m C$. If not, there must exist $i$ and $j$ such that $d:=j-i>C$ and the uniform dimension of $A / I_{i}$ is the same as the uniform dimension of $A / I_{j}$. Thus there exists a right ideal $J$ such that $I_{\ell} \cap J=(0)$ for $i \leq \ell \leq j$ and $I_{\ell}+J$ is essential (that is, it contains a regular element) for $i \leq \ell \leq j$. Let

$$
J_{\ell}=I_{i+\ell}+J
$$

for $0 \leq \ell \leq d$.

By Lemma 2.3 there are positive integers $m_{1}, m_{2}, \ldots, m_{d}$ such that

$$
\operatorname{dim}\left(V^{n} \cap J_{j} / V^{n} \cap J_{j-1}\right) \geq n-m_{j}
$$


for all $n$. Hence

$$
\begin{aligned}
\operatorname{dim}\left(V^{n}\right) & \geq \operatorname{dim}\left(V^{n} \cap J_{d}\right) \\
& =\operatorname{dim}\left(V^{n} \cap J_{0}\right)+\sum_{i=1}^{d} \operatorname{dim}\left(V^{n} \cap J_{i} / V^{n} \cap J_{i-1}\right) \\
& \geq \operatorname{dim}\left(V^{n} \cap J_{0}\right)+\sum_{i=1}^{d}\left(n-m_{i}\right) \\
& \geq \operatorname{dim}\left(V^{n} \cap J_{0}\right)+d n-\left(m_{1}+\cdots+m_{d}\right) .
\end{aligned}
$$

Thus

$$
\operatorname{dim}\left(V^{n}\right)-\operatorname{dim}\left(V^{n} \cap J_{0}\right) \geq d n-\left(m_{1}+\cdots+m_{d}\right)
$$

for all $n$. But $J_{0}$ contains a regular element and hence for infinitely many $n$ we have

$$
\operatorname{dim}\left(V^{n}\right)-\operatorname{dim}\left(V^{n} \cap J_{0}\right) \leq C n
$$

by Lemma 2.2. Thus $d \leq C$, since $m_{1}, \ldots, m_{d}$ are fixed. It follows that $A$ is right noetherian. By looking at the opposite ring, we see $A$ is also left noetherian. To see that $A$ has Krull dimension 1, note that if $J \supset I$, and $I$ is nonzero, then any chain of submodules of $J / I$ in which containments are proper must have length at most $C=m C(I, V)$. In particular, $J / I$ is artinian. Hence $A$ has Krull dimension 1. By a result of Stafford, every right and left ideal of $A$ is generated by at most two elements.

Proof of Corollary 1.2. This follows immediately from Theorem 1.1 and Proposition 4.13 of Krause and Lenagan 4 .

Proof of Corollary 1.3. By a result of Irving and Small [3], a simple algebra of quadratic growth is right Goldie if and only if it is left Goldie. If such an algebra is either right or left noetherian, then it is Goldie and so it is noetherian on both sides.

\section{Concluding Remarks}

We note that the finitely generated hypothesis is necessary in Theorem 1.1 to ensure we obtain noetherianity. Let

$$
B=k\left[t, t^{1 / 2}, t^{1 / 4}, \ldots\right],
$$

and let $A=B[x ; \delta]$, where $\delta$ is differentiation with respect to $t$. Then $A$ is a free $B$-module and since $B$ is not noetherian, neither is $A$. We note that $A$ is a simple domain and any finitely generated subalgebra of $A$ has quadratic growth.

We note that prime Goldie algebras of quadratic growth need not be noetherian in general. To see this, let

$$
A=\left(\begin{array}{cc}
k\left[x, y, y^{-1}\right] & k\left[x, y, y^{-1}\right] x \\
k\left[x, y, y^{-1}\right] & k[y]+k\left[x, y, y^{-1}\right] x
\end{array}\right) .
$$

We note that $A$ is a finitely generated non-noetherian prime Goldie $k$-algebra (cf. McConnell and Robson [5, 13.10.2]). It is straightforward to check that $A$ has quadratic growth. A non-PI example of a non-noetherian finitely generated simple Goldie algebra of quadratic growth can be constructed over any field $k$ that is not algebraic over a finite field by using $k_{q}\left[x, y, y^{-1}\right]$ in $A$ instead of $k\left[x, y, y^{-1}\right]$, where $k_{q}\left[x, y, y^{-1}\right]$ is a localization of the quantum plane; that is, $x y=q y x$, with $q$ nonzero 
and not a root of unity. This type of non-PI construction cannot be made over a finite field since every nonzero element of a finite field is a root of unity and so the resulting algebra is PI. Smoktunowicz 6] has shown that in many cases, a finitely generated prime algebra over a finite field satisfies a polynomial identity. We thus make the following conjecture.

Conjecture 3.1. Let $k$ be a finite field and let $A$ be a finitely generated $k$-algebra of quadratic growth. Then $A$ is not simple.

Artin and Stafford showed in the course of their description of graded domains of GK dimension two that all non-PI graded domains of quadratic growth that are generated in degree 1 are noetherian. It is an interesting question as to whether a similar description of the quotient division algebras of simple domains of quadratic growth can be given. More specifically, can it be shown that a simple domain of quadratic growth is birationally isomorphic to a graded domain of quadratic growth or to the ring of differential operators on a curve? Artin and Stafford also made the conjecture that a "gap" should exist in the possible GK dimensions that a graded domain can attain. Smoktunowicz [7] proved this conjecture. It would be interesting if a similar gap result could be attained for simple domains; that is, can it be shown that there are no finitely generated simple domains of GK dimension strictly between 2 and 3 ?

We also note that the techniques used in the proof of Theorem 1.1 do not extend to higher GK dimension. Nevertheless, we are unaware of a counter-example for algebras of GK dimension 3. We thus pose the following questions.

Question 3.2. Does there exist a non-noetherian finitely generated simple domain of GK dimension 3 ?

Question 3.3. Is the Krull dimension of a finitely generated simple noetherian algebra of GK dimension 3 at most 2 ?

\section{ACKNOWLEDGMENTS}

I thank the anonymous referee for many helpful comments and suggestions.

\section{REFERENCES}

1. M. Artin and J.T. Stafford, Noncommutative graded domains with quadratic growth. Invent. Math. 122 (1995), no. 2, 231-276. MR1358976 (96g:16027)

2. W. Borho and H. Kraft, Über die Gelfand-Kirillov-Dimension. Math. Ann. 220 (1976), no. 1, 1-24. MR0412240 (54:367)

3. R. S. Irving and L. W. Small, The Goldie conditions for algebras with bounded growth. Bull. London Math. Soc. 15 (1983), no. 6, 596-600. MR720748 (85a:16017)

4. G. R. Krause and T. H. Lenagan, Growth of algebras and Gelfand-Kirillov dimension. Revised edition. Graduate Studies in Mathematics, 22. American Mathematical Society, Providence, RI, 2000. MR1721834 (2000j:16035)

5. J. C. McConnell and J. C. Robson, Noncommutative Noetherian Rings. Wiley-Interscience, New York, 1987. MR 934572 (89j:16023)

6. A. Smoktunowicz, On structure of domains with quadratic growth. J. Algebra 289 (2005), no. 2, 365-379. MR2142377(2006a:16030) 
7. A. Smoktunowicz, There are no graded domains with GK dimension strictly between 2 and 3. Invent. Math. 164 (2006), 635-640. MR.2221134(2007b:16047)

8. J. T. Stafford, Completely faithful modules and ideals of simple Noetherian rings. Bull. London Math. Soc. 8 (1976), no. 2, 168-173. MR0399159 (53:3010)

Department of Mathematics, Simon Fraser University, 8888 University Drive, Burnaby, British Columbia, Canada, V5A 1S6

E-mail address: jpb@math.sfu.ca 